\title{
A Novel Method for Partially Adaptive Broadband Beamforming
}

\author{
Wei Liu, Stephan Weiss*, and Lajos Hanzo \\ Communications Research Group \\ Dept. of Electronics \& Computer Science \\ University of Southampton, Southampton SO17 1BJ, UK \\ Tel. +4402380 597645, Fax +4402380594508 \\ $\{$ w. liu, s.weiss, 1 .hanzo\}@ecs. soton.ac.uk
}

\begin{abstract}
In this paper, a novel subband-selective generalized sidelobe canceller (GSC) for partially adaptive broadband beamforming is proposed. The blocking matrix of the GSC is constructed such that its columns constitute a series of bandpass filters, which select signals with specific angles of arrival and frequencies. This results in bandlimited spectra of the blocking matrix outputs, which is further exploited by a subbands decomposition prior to running independent unconstrained adaptive filters in each non-redundant subband. We discuss the design of both the blocking matrix using a genetic algorithm an efficient sum-of-power-of-two coefficient format and the filter bank for the subsequent subband decomposition. By these steps, the computational complexity of our subband-selective GSC is greatly reduced compared to other partially adaptive GSC schemes, while performance is comparable or even enhanced due to subband decorrelation, as simulations indicate.
\end{abstract}

Keywords. broadband beamforming, subband processing, adaptive filters, low complexity implementation

* Corresponding Author 


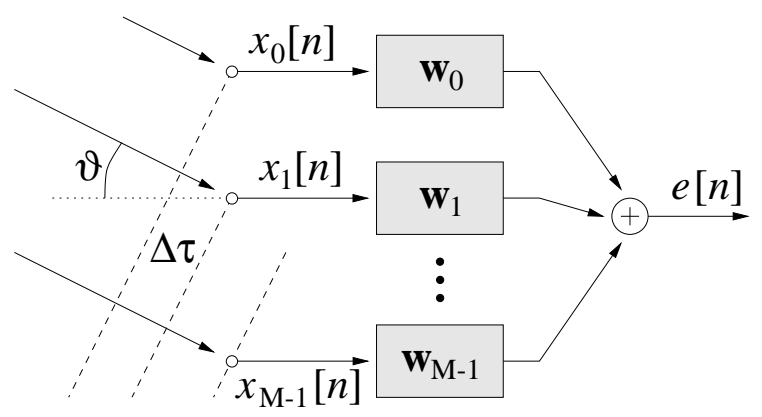

Fig. 1. A signal impinging from an angle $\vartheta$ onto a beamformer with $M$ sensors.

\section{INTRODUCTION}

Adaptive beamforming has found many applications in various areas ranging from sonar and radar to wireless communications. It is based on a technique where, by adjusting the weights of a sensor array with attached filters, a prescribed spatial and spectral selectivity is achieved. Thus, signals arriving from unwanted directions of arrival (DOA) may be suppressed or enhanced by destructive or constructive interference in the beamfomer, as illustrated in Fig. 1. To perform beamforming with high interference rejection and resolution, arrays with a large number of sensors and filter coefficients have to be employed and the computational burden of a fully adaptive processor thus becomes considerable. A popular way to reduce the computational complexity are partially adaptive beamformers, which employ a subset of available degrees of freedom (DOF) in the filter weight update process at the expense of a somewhat reduced performance [1].

Partially adaptive techniques have been studied widely and many ideas have been proposed such as the weight reduction transformation [2] and the eigencanceler [3]. In this paper, with a generalized sidelobe canceller (GSC) [4], [5] as the underlying structure, we propose a novel design for partially adaptive beamformers. It is based on the design of the blocking matrix constituting a series of bandpass filters. These filters separate the impinging signals into components of different DOA and frequencies. Furthermore, subband techniques are employed to further decompose the output of the blocking matrix, yielding a low-cost implementation and additionally providing an effective decorrelation of the input to the adaptive process. 
The paper is organised as follows. Section II briefly reviews GSC based fully and partially adaptive broadband beamforming. The novel subband-selective blocking matrix is introduced in Sec. III, and the subsequent subband decomposition process is described in Sec. IV. The design of both the blocking matrix via a genetic algorithm (GA) and the subband decomposition are addressed in Sec. V. Finally, simulations underlining the benefit of the proposed method are discussed in Sec. VI and conclusions drawn in Sec. VII.

\section{Partially Adaptive Generalised Sidelobe Canceller}

A linearly constrained minimum variance (LCMV) beamformer performs the minimization of the variance or power of the output signal with respect to some given spatial and spectral constraints. For a beamformer with $M$ sensors and $J$ filter taps following each sensor, the output $y[n]$ can be expressed as:

$$
y[n]=\mathbf{w}^{\mathrm{H}} \cdot \mathbf{x}_{n}
$$

where coefficients and input sample values are defined as

$$
\begin{aligned}
& \mathbf{w}=\left[\begin{array}{llll}
\mathbf{w}_{0}^{\mathrm{T}} & \mathbf{w}_{1}^{\mathrm{T}} & \ldots & \mathbf{w}_{J-1}^{\mathrm{T}}
\end{array}\right]^{\mathrm{H}} \\
& \mathbf{w}_{l}=\left[\begin{array}{llll}
w_{0}[l] & w_{1}[l] & \ldots & w_{M-1}[l]
\end{array}\right]^{\mathrm{T}} \\
& \mathbf{x}_{n}=\left[\begin{array}{llll}
\tilde{\mathbf{x}}_{n}^{\mathrm{T}} & \tilde{\mathbf{x}}_{n-1}^{\mathrm{T}} & \ldots & \tilde{\mathbf{x}}_{n-J+1}^{\mathrm{T}}
\end{array}\right]^{\mathrm{T}}
\end{aligned}
$$

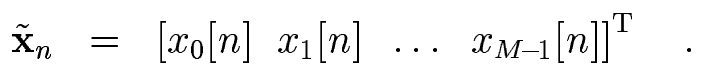

The data vector $\tilde{\mathbf{x}}_{n}$ is a time slice as given in Fig. 1 , where a coefficient $w_{m}[l]$ belongs to the $m$ th filter sitting at tap position $l$. The LCMV problem can be formulated as

$$
\min _{\mathbf{w}} \mathbf{w}^{\mathrm{H}} \mathbf{R}_{x x} \mathbf{w} \quad \text { subject to } \quad \mathbf{C}^{\mathrm{H}} \mathbf{w}=\mathbf{f}
$$

where $\mathbf{R}_{x x}$ is the covariance matrix of observed array data in $\mathbf{x}_{n}, \mathbf{C} \in \mathbb{C}^{M J \times S J}$ is a constraint matrix and $\mathbf{f} \in \mathbb{C}^{S J}$ is the constraining vector. The constraint matrix here imposes derivative 


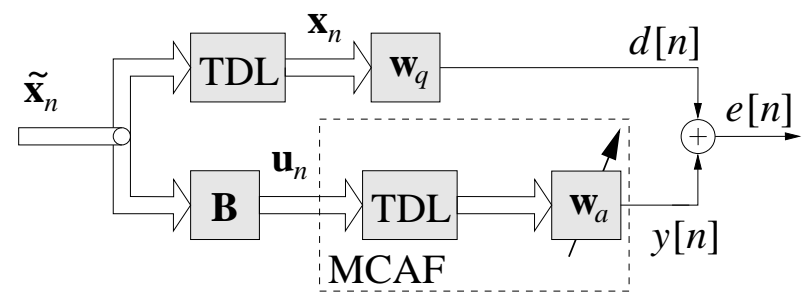

Fig. 2. GSC with derivative constraint blocking matrix B; with tapped-delay lines (TDL) the GSC optimisation is unconstrained and can be performed by a multichannel adaptive filter (MCAF).

constraints of order $S-1$,

$$
\mathbf{C}=\left[\hat{\mathbf{C}}_{0} \ldots \hat{\mathbf{C}}_{S-1}\right] \quad \text { with } \quad \hat{\mathbf{C}}_{i}=\left[\begin{array}{ccc}
\mathbf{c}_{i} & & \mathbf{0} \\
& \ddots & \\
\mathbf{0} & & \mathbf{c}_{i}
\end{array}\right] \in \mathbb{C}^{M J \times J}
$$

with $\mathbf{c}_{i}=\left[\begin{array}{llll}\left(-m_{0}\right)^{i} & \left(1-m_{0}\right)^{i} & \cdots & \left(M-1-m_{0}\right)^{i}\end{array}\right]^{\mathrm{T}}$. The phase origin $m_{0}$ defines the reference point on the $M$-element linear array. Without loss of generality, for convenience we assume $m_{0}=0$ in the following.

The constrained optimisation of the LCMV problem in (6) can be conveniently solved by means of a GSC. The GSC performs a projection of the data onto an unconstrained subspace by means of a blocking matrix $\mathbf{B}$ and a quiescent vector $\mathbf{w}_{\mathrm{q}}$. Thereafter, standard unconstrained optimisation algorithms such as least mean square (LMS) or recursive least squares (RLS) algorithms can be invoked [6]. Fig. 2 shows the principle of a GSC, where a desired signal $d[n]$ is obtained via $\mathbf{w}_{\mathrm{q}}$,

$$
d[n]=\mathbf{w}_{\mathrm{q}}^{\mathrm{H}} \cdot \mathbf{x}_{n} \quad \text { with } \quad \mathbf{w}_{\mathrm{q}}=\mathbf{C}\left(\mathbf{C}^{\mathrm{H}} \mathbf{C}\right)^{-1} \mathbf{f}
$$

The input vector $\mathbf{u}_{n} \in \mathbb{C}^{L}$ to the optimisation process is obtained by $\mathbf{u}_{n}=\mathbf{B}^{\mathrm{H}} \tilde{\mathbf{x}}_{n}$, whereby the blocking matrix must satisfy

$$
\tilde{\mathbf{C}}^{\mathrm{H}} \mathbf{B}=\mathbf{0} \quad \text { where } \quad \tilde{\mathbf{C}}=\left[\begin{array}{lll}
\mathbf{c}_{0} & \cdots & \mathbf{c}_{S-1}
\end{array}\right] \in \mathbb{C}^{M \times S}
$$

with

$$
\begin{aligned}
\mathbf{B} & =\left[\begin{array}{llll}
\mathbf{b}_{0} & \mathbf{b}_{1} & \ldots & \mathbf{b}_{L-1}
\end{array}\right] \quad \text { and } \\
\mathbf{b}_{l} & =\left[\begin{array}{llll}
b_{l}[0] & b_{l}[1] & \ldots & b_{l}[M-1]
\end{array}\right]^{\mathrm{H}} .
\end{aligned}
$$


As only (9) has to be satisfied, the dimension of $\mathbf{B}$ can be selected arbitrarily with $L \leq M-S$. For $L<M-S$, the resulting beamformer has a reduced number of DOFs and is termed a partially adaptive GSC [1], offering reduced complexity traded off against a somewhat inferior performance. In the next section, we will sacrifice some DOFs to obtain a particular form of the blocking matrix $\mathbf{B}$.

\section{Spatially Selective Blocking Matrix Via Subbands}

The aim is to find a blocking matrix $\mathbf{B}$ that offers spatial selectivity. Considering a unity amplitude complex input wave with angular frequency $\omega$ and DOA angle $\vartheta$, the received phasor vector at the sensor array, $\underline{X}$, is

$$
\underline{X}=\left[\begin{array}{llll}
1 & e^{-j \omega \Delta \tau} & \cdots & e^{-j \omega(M-1) \Delta \tau}
\end{array}\right]^{T} \quad \text { with } \quad \Delta \tau=\frac{d}{c} \sin (\vartheta)
$$

Referring to Fig. 1, the waveform impings with a time delay $\Delta \tau$ on adjacent sensors separated by $d$ in a medium with propagation speed $c$. The distance $d$ is the spatial sampling period, which relates to an angular sampling wavenumber $\kappa_{\mathrm{s}}=2 \pi / d$. If spatial and temporal sampling are related by $\kappa_{\mathrm{s}}=\omega_{\mathrm{s}} / c$, then with the normalised angular sampling frequency $\Omega=2 \pi \omega / \omega_{\mathrm{s}}$

$$
\underline{X}=\left[\begin{array}{llll}
1 & e^{-j \Omega \sin \vartheta} & \cdots & e^{-j(M-1) \Omega \sin \vartheta}
\end{array}\right]^{\mathrm{T}}
$$

Hence the $l$ th output of the block $\mathbf{B}$ in Fig. 2 can be denoted, with the substitution $\Psi=$ $\Omega \sin \vartheta$

$$
U_{l}=\mathbf{b}_{l}^{\mathrm{H}} \cdot \underline{X}=\sum_{m=0}^{M-1} b_{l}[m] \cdot e^{-j m \Psi}=B_{l}\left(e^{j \Psi}\right)
$$

whereby $B_{l}\left(e^{j \Psi}\right) \bullet-\circ b_{l}[m]$ is a Fourier pair.

According to (13), the columns of the blocking matrix $\mathbf{B}$ can be regarded as a set of spatial filters. If the beamformer is constrained to receive the signal of interest from broadside, then the blocking matrix has to suppress any component impinging from $\vartheta=0$. Therefore, at $\Psi=0$ the response of the $\mathbf{b}_{l}$ has to be zero. For an interesting case, we arrange 


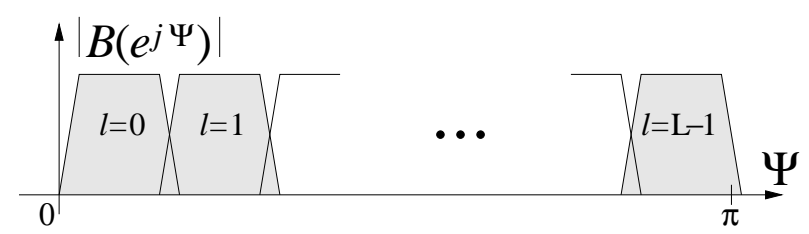

Fig. 3. Characteristics of the $L$ spatial filters contained in $\mathbf{B}$.

the design of the $\mathbf{b}_{l}$ to yield spatial bandpass filters of the type

$$
B_{l}\left(e^{j \Psi}\right)= \begin{cases}1 & \text { for } \Psi \in\left[\Psi_{\text {lower }} ; \Psi_{\text {upper }}\right] \\ 0 & \text { otherwise }\end{cases}
$$

on the interval $\Psi \in[0 ; \pi]$. The arrangement is depicted in Fig. 3. All values apart from $\Psi=0$ have to be covered to satisfy the constraints. The appeal is - if signals imping only from a number of angles - that only some outputs of the blocking matrix and therefore some branches of the multichannel adaptive filter will contain significant contributions. Hence, a design of the blocking matrix columns according to Fig. 3 will lead to a spatial decomposition or decorrelation of the array data, and may therefore be expected to improve the convergence characteristics of an LMS-type adaptive algorithm employed for updating the MCAF. An additional advantage of the spatial filtering will be discussed in Sec. IV when combining $\mathbf{B}$ with a successive spectral decomposition.

In reality, the bandpass filters $B_{l}\left(e^{j \Psi}\right)$ will not be ideal and hence an overlap and finite transition bands have to be permitted. However, a better design quality can be attained by reducing the number of columns, $L$, below the limit $M-S$, thus yielding a partially adaptive beamformer, sacrificing some DOFs in the beamformer. A design method for $\mathbf{B}$ will be described in Sec. V-A.

\section{Spectral Selectivity Via Subband Processing}

The previous Sec. III has introduced a spatial decorrelation of the input signal. Now, a spectral decomposition is applied to the $L$ outputs of the blocking matrix $\mathbf{B}$ in Fig. 2 analogously to well-known subband adaptive filtering techniques [7]. Besides their prewhitening property, such subband methods offer reduced computational cost and yield structures that lend themselves to parallel implementations. 


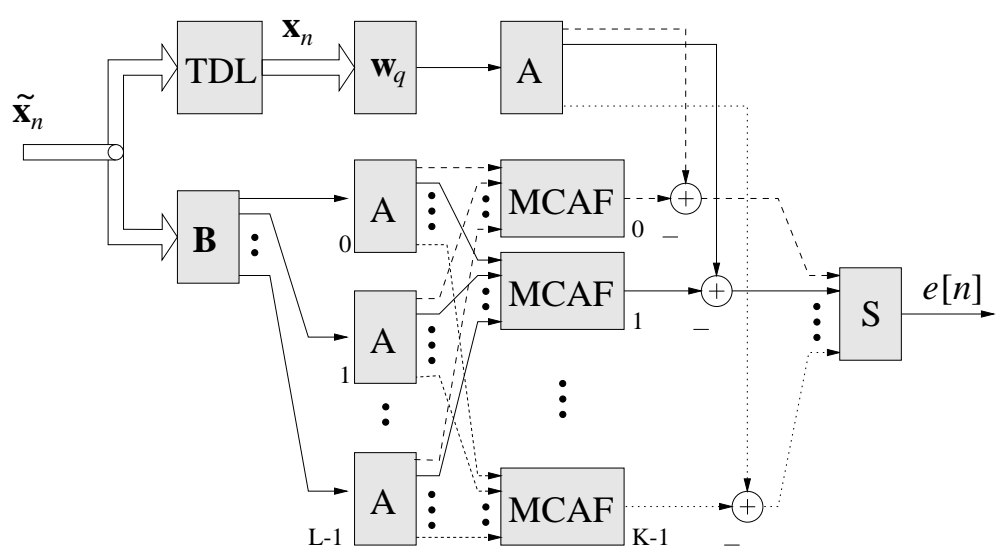

Fig. 4. A setup of suband decomposition following the blocking matrix; an independent MCAF is applied to each of the subband.

Subband decompositions for adaptive filter applications are commonly based on oversampled modulated filter banks (OSFB), dividing an input signal into $K$ frequency bands decimated by a factor $N$. Due to oversampling, i.e. $N<K$, a low alias level in the subband signals can be achieved. This is important since aliasing will limit the performance of an SAF system [8]. Due to its lower update rate and fewer coefficients to represent an impulse response of a given length, the subband implementation only necessitates $K / N^{2}\left(K / N^{3}\right)$ of the operations required for a fullband adaptive algorithm with a complexity of $\mathcal{O}\left(L_{\mathrm{a}}\right)$ $\left(\mathcal{O}\left(L_{\mathrm{a}}^{2}\right)\right)$, where $L_{\mathrm{a}}$ is the total number of coefficients in the fullband realisation [9].

When applying SAF methods to the MCAF in the derivative-constrained GSC in Fig. 2, the subband setup as shown in Fig. 4 arises. There, the blocks labelled A perform an OSFB analysis operations, splitting the signal into $K$ frequency bands each running at an $N$ times lower sampling rate than the fullband input to the block. Within each subband, an independent MCAF is operated, and a synthesis filter bank, labelled S, recombines the different subsystem outputs to a fullband beamformer output $e[n]$.

An interesting property of the subband splitting emerges when considering the spatial decomposition by the blocking matrix $\mathbf{B}$ as introduced in Sec. III. Considering $\Omega=\Psi / \sin \vartheta$, the spatial filters $\mathbf{b}_{l}$ also affect the spectrum of the $L$ output branches of $\mathbf{B}$ :

$$
B_{l}\left(e^{j \Omega}\right)= \begin{cases}1 & \text { for } \mu \in\left[\Psi_{\text {lower }} ; \pi\right] \\ 0 & \text { otherwise }\end{cases}
$$


based on the definition of $B\left(e^{j \Psi}\right)$ in (14). Note that with increasing $l$, these spatial filters are associated with a tighter and tighter highpass spectrum. Therefore, if sufficiently selective column vectors $\mathbf{b}_{l}$ can be designed, then the dimensionality of the MCAFs can be reduced accordingly by omitting unnecessary lowpass bands from processing. For example, the last output $(L-1)$ of the blocking matrix in Fig. 4 may only contain the ultimate highpass component, and so forth. Hence e.g. the first $(k=0)$ MCAF would be a single channel adaptive filter drawing its low frequency input solely from the first branch of $\mathbf{B}$. The second $(k=1)$ MCAF block in Fig. 4 will most likely only cover some of the lower branches of B, while finally only the last MCAF $(K-1)$ consists of $L-1$ non-sparse channels. Thus, a channel reduction in the MCAFs is achieved yielding a considerably reduced complexity. This characteristic underlines the advantage of a combined spatial/spectral subband selection by subband processing in both the spatial and spectral domain. Therefore, the SAF beamforming structure in Fig. 4 is named a subband-selective GSC.

\section{IMPLEMENTATION AND DESIGN}

\section{A. SOPOT Design of the Blocking Matrix}

In our subband-selective GSC, the blocking matrix plays a central role. In order to obtain a design that fulfills both the desired spatial bandpass characteristic w.r.t. $\Psi$ and achieves a low computational complexity, a GA is employed which seeks a realisation of $\mathbf{B}$ with sum of power of two (SOPOT) coefficients [10], [11]. The arithmetic for $\mathbf{B}$ can therefore be implemented by simple shifts and additions.

From (9), we obtain an expression for the constraints to be fulfilled by the lth column filter of the blocking matrix when considering $S-1$ order derivative constraints,

$$
\sum_{m=0}^{M-1} m^{i} \cdot b_{l}[m]=0 \quad, \quad \text { for } \quad i=0(1) S-1, \quad l=0(1) L-1
$$

Under the constraint (16), a minimax optimisation of the spatial filters $\mathbf{b}_{l}$ can be formulated,

$$
\mathbf{b}_{l}=\arg \min _{\mathbf{b}_{l}}\left\|B_{l}\left(e^{j \Psi}\right)-\tilde{B}_{l}\left(e^{j \Psi}\right)\right\|_{\infty} \quad, \quad \Psi \in[0 ; \pi]
$$


whereby the response $B_{l}\left(e^{j \Psi}\right)$ is as defined in $(13)$ and $\tilde{B}_{l}\left(e^{j \Psi}\right)$ is the desired spatial response of the $l$ th filter.

If (16) is used to directly fix $S$ parameters, the optimisation can be performed over the remaining $M-S$ coefficients. As an example, let us consider the case of first order derivative constraints, $S=2$ :

$$
\begin{aligned}
& b_{l}[0]=-\sum_{m=1}^{M-1} b_{l}[m] \quad \text { for } \quad i=0 \\
& b_{1}[1]=-\sum_{m=2}^{M-1} m \cdot b_{l}[m] \quad \text { for } \quad i=1
\end{aligned}
$$

Note, that the optimisation problem has been transformed into an unconstrained optimisation over the remaining coefficients $b_{l}[2]$ to $b_{l}[M-1]$, which is straightforward to solve by means of a GA.

In order to obtain an efficient implementation and also maintain a reasonable performance space over which the GA performs its optimisation, coefficients are constrained to be SOPOT. Therefore, a coefficient $b_{l}[m]$ can be represented as

$$
b_{l}[m]=\sum_{p=0}^{P[l, m]-1} a_{p}[l, m] \cdot 2^{L_{p}[l, m]} \quad \text { with } \quad a_{p}[l, m] \in\{-1 ; 1\} \quad, \quad L_{p}[l, m] \in\{-Q(1) Q\}
$$

whereby $P \geq P[l, m]$ is a limit for the number of SOPOT terms and $Q$ is the maximum wordlength of the number representation. Normally, $P$ is limited to a small number. The coefficients resulting finally from the GA optimisation can be implemented by binary shift and add operations, hence avoiding multiplications.

Example. Fig. 5 gives a GA design result for the blocking matrix with $M=16$ sensors, $S=2$ order constraints. A partially adaptivity was assumed with $L=8$. Displayed are the $B_{l}\left(e^{j \Psi}\right)$, which exhibit a reasoably good bandpass characteristic. The maximum number of SOPOT terms, $P$, was set to three. 


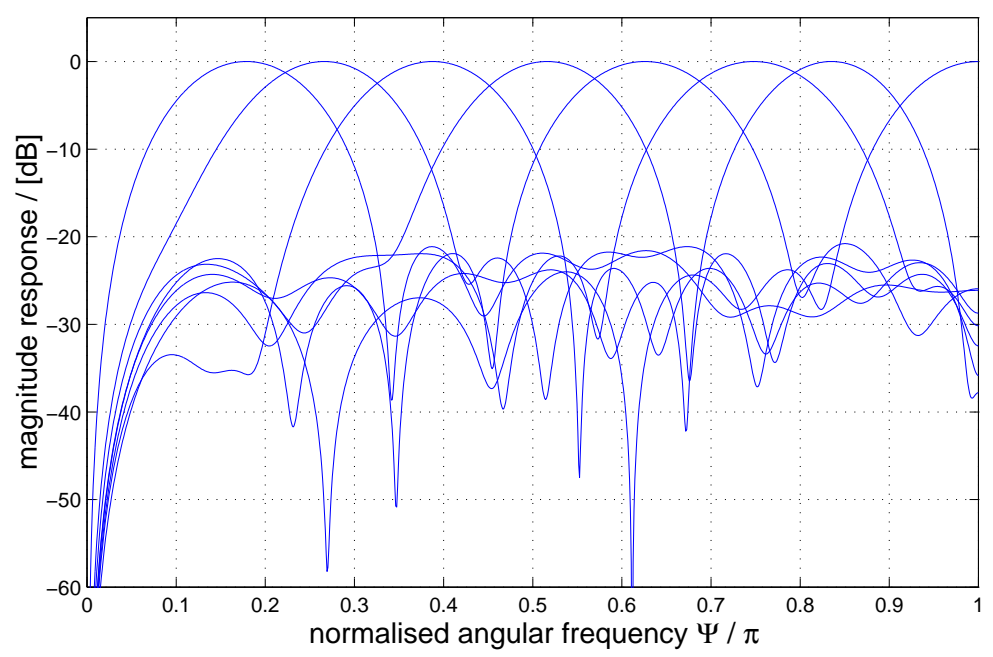

Fig. 5. Characteristics of the blocking matrix columns using a GA design with SOPOT realisation.

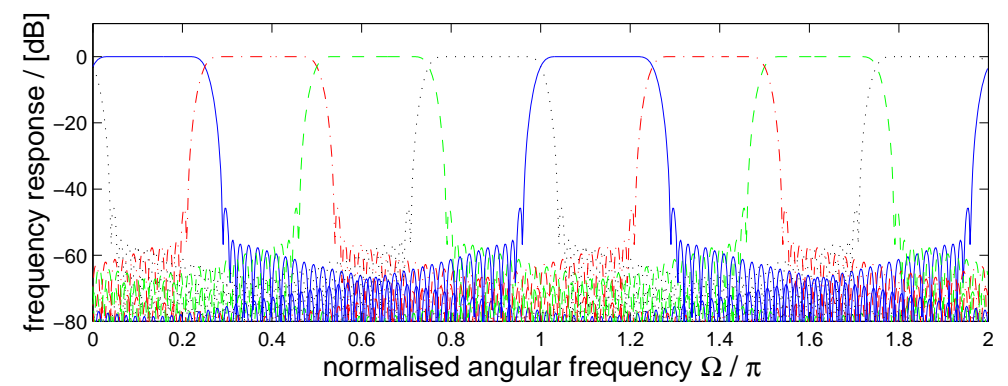

Fig. 6. Frequency response of a $K=8$ channel filter bank decimated by $N=6$.

\section{B. Filter Bank Implementation and Design}

For the spectral decomposition, OSFBs permit a computationally inexpensive filter bank implementation [12]. For a given channel number $K$, decimation ratio $N$, and the filter length for the OSFB, an iterative least squares design can be invoked, which allows a tradeoff between SAF performance limiting factor such as the aliasing level in the subbands, and the error in perfect reconstruction [13].

A design example for $K=8, N=6$ and a filter length of 96 coefficients is given Fig. 6 . The filters are modulated from a prototype lowpass filter by means of a generalised discrete Fourier transform (GDFT), whereby 'generalised' refers to the inclusion of offset terms into the DFT [14]. The upper 4 bands are redundant for real valued OSFB input, and can therefore be omitted in this case. 


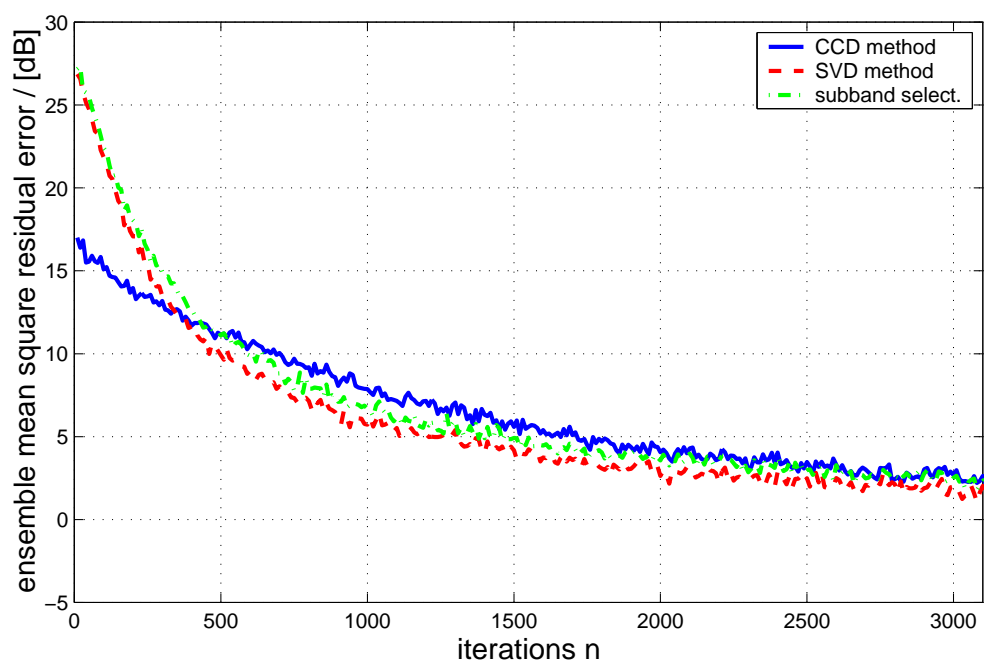

Fig. 7. Learning curves for fullband realisations of different beamforming structures.

\section{Simulations and Results}

The following simulations are based on a beamformer setup as in Fig. 1 with $M=16$ sensors and followed TDLs of $J=60$ taps. The GSC is constrained by first order derivatives $(S=2)$ to receive a wideband signal of interest from broadside $\left(\vartheta=0^{\circ}\right)$. The beamformer should adaptively suppress a broadband interference signal covering the frequency interval $\Omega=[0.25 \pi ; 0.75 \pi]$ from $\vartheta=15^{\circ}$ and with a signal-to-interference ratio (SIR) of $-24 \mathrm{~dB}$. Additionally, all sensors receive spectrally and spatially uncorrelated noise at $20 \mathrm{~dB}$ SNR.

In a first set of simulations, we apply only a spatial decomposition as described in Sec. III. The resulting partially adaptive GSC as shown in Fig. 2 is implemented with a reduced blocking matrix $\mathbf{B} \in \mathbb{R}^{L \times M}$ with $L=8$. The characteristic of $\mathbf{B}$ is the one given in the design example in Fig. 5. In Fig. 7, this system is compared with two other design methods for blocking matrices, i.e. the cascaded columns of difference (CCD) methods [15] and a singular value decomposition (SVD) to find the nullspace of the constraint matrix $\tilde{\mathbf{C}}[5]$. The depicted performance measure is the mean square valued of the residual error, i.e. the beamformer output $e[n]$ minus the signal of interest impinging from broadside. Both CCD and SVD structures are fully adaptive beamformers. The MCAFs in the different beamformers are updated by a normalised LMS algorithm with a step size of 0.25 [6]. As evident from Fig. 7, the CCD system gives an initially faster convergence, but all three 


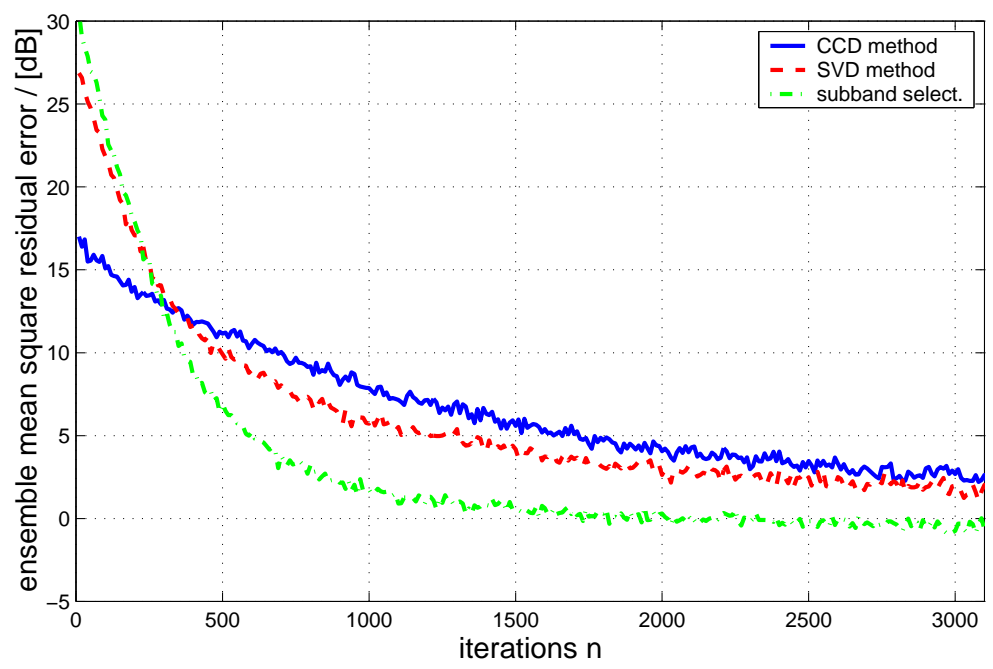

Fig. 8. Learning curves for different beamforming structures with spectral subband decomposition.

structures provide a similar performance over a longer period of adaptation.

For a second set of simulations, additional spectral subband splitting according to Sec. IV is applied subsequent to the subband-selective blocking matrix design, as shown in Fig. 4. Each of the TDL signals following the blocking matrix and the reference signal $d[n]$ are divided into $K=8$ subbands by the OSFB of Fig. 6 with decimation ratio $N=6$. This in general results in a lower computational complexity compared to the previous fullband implementations, both through the subband approach itself and through discarding unrequired subbands, c.f. Sec. IV. Inspecting the resulting mean square error curves in Fig. 8, the system with both subband selective blocking matrix and spectral subband decomposition substantially outperforms the other realisations in terms of convergence speed by avoiding slow modes of adaptation. This is due to the decorrelating effect of both the blocking matrix and the OSFB analysis filter banks.

\section{ViI. Conclusions}

A subband-selective generalized sidelobe canceller for partially adaptive broadband beamforming has been proposed. In this beamforming structure, the column vectors of the blocking matrix constitute a series of bandpass filters, which decompose the impinging signals into components of specific angles of arrival and frequencies. This is followed by a spectral 
subband decomposition, which further decorrelates the beamformer input. Due to the particular characteristics of the blocking matrix, some of the spectral lowpass subbands do not require to be processed. Moreover, in order to further reduce the computational complexity of the blocking matrix, we have proposed a GA design method for the subband-selective blocking matrix such that its elements are of a SOPOT form.

This permits a considerably reduced computational complexity. As demonstrated in a number of simulations, our subband-selective approach has the additional benefit of faster convergence for LMS-type adaptive algorithms employed in the GSC.

\section{REFERENCES}

[1] D. J. Chapman, "Partial Adaptivity for Large Arrays," IEEE Transactions on Antennas and Propagation, vol. 24, no. 9, pp. 685-696, September 1976.

[2] B. D. Van Veen and R. A. Roberts, "Partially Adaptive Beamforming Design via Output Power Minimization," IEEE Transactions on Acoustics, Speech, and Signal Processing, vol. 35, pp. 1524-1532, 1987.

[3] B. D. Van Veen, "Eigenstructure Based Partially Adaptive Array Design," IEEE Transactions on Antennas and Propagation, vol. 36, no. 1, pp. 357-362, January 1988.

[4] L. J. Griffith and C. W. Jim, "An Alternative Approach to Linearly Constrained Adaptive Beamforming," IEEE Transactions on Antennas and Propagation, vol. 30, no. 1, pp. 27-34, January 1982.

[5] K. M. Buckley and L. J. Griffith, "An Adaptive Generalized Sidelobe Canceller with Derivative Constraints," IEEE Transactions on Antennas and Propagation, vol. 34, no. 3, pp. 311-319, March 1986.

[6] S. Haykin, Adaptive Filter Theory, Prentice Hall, Englewood Cliffs, 2nd edition, 1991.

[7] W. Kellermann, "Analysis and Design of Multirate Systems for Cancellation of Acoustical Echoes," in Proc. IEEE International Conference on Acoustics, Speech, and Signal Processing, New York, 1988, vol. 5, pp. 2570-2573.

[8] S. Weiss, R. W. Stewart, A. Stenger, and R. Rabenstein, "Performance Limitations of 
Subband Adaptive Filters," in European Signal Processing Conference, Rodos, Greece, September 1998, vol. III, pp. 1245-1248.

[9] S. Weiss, R. W. Stewart, M. Schabert, I. K. Proudler, and M. W. Hoffman, "An Efficient Scheme for Broadband Adaptive Beamforming," in Asilomar Conference on Signals, Systems, and Computers, Monterey, CA, November 1999, vol. I, pp. 496-500.

[10] K. S. Tang, K. F. Man, S. Kwong, and Q. He, "Genetic Algorithms and Their Applications," IEEE Signal Processing Magazine, pp. 22-37, November 1996.

[11] W. Liu, S. C. Chan, and K. L. Ho, "Low-Delay Perfect Reconstruction Two-Channel FIR/IIR Filter Banks and Wavelet Bases with SOPOT Coefficients," in Proc. IEEE International Conference on Acoustics, Speech, and Signal Processing, Istanbul, Turkey, June 2000.

[12] S. Weiss and R. W. Stewart, "Fast Implementation of Oversampled Modulated Filter Banks," IEE Electronics Letters, vol. 36, no. 17, pp. 1502-1503, August 2000.

[13] M. Harteneck, S. Weiss, and R. W. Stewart, "Design of Near Perfect Reconstruction Oversampled Filter Banks for Subband Adaptive Filters," IEEE Transactions on Circuits \& Systems II, vol. 46, no. 8, pp. 1081-1086, August 1999.

[14] R. E. Crochiere and L. R. Rabiner, Multirate Digital Signal Processing, Prentice Hall, Englewood Cliffs, NJ, 1983.

[15] N. K. Jablon, "Steady State Analysis of the Generalized Sidelobe Canceler by Adaptive Noise Canceling Techniques," IEEE Transactions on Antennas and Propagation, vol. 34, no. 3, pp. 330-337, March 1986. 